\title{
Ventilación no invasiva como tratamiento de la insuficiencia respiratoria aguda en Pediatría
}

\author{
Francisco Prado $A^{1,3}$, María Adela G odoy $R^{2}$, \\ Marcela Godoy P2, María Lina Boza $C^{3}$. \\ Pediatric non-invasive ventilation \\ for acute respiratory failure \\ in an Intermediate Care U nit
}

Background: Pediatric noninvasive ventilation (NIV) is infrequently used for acute respiratory failure (ARF), BiPAP/CPAP applied through nasal mask can be attempted if strict selection rules are defined. Aim: To evaluate the outcome of NIV in a Pediatric Intermediate Care Unit. Material and methods: The medical records of 14 patients (age range 1 month-13 years, six female), who participated in a prospective protocol of NIV from January to October 2004, were reviewed. Oxygen therapy, delivered through a reservoir bag attached to the ventilation circuit, was used to maintain $\mathrm{SaO}_{2}$ over $90 \%$. Results: The main indication of BiPAP, in $80 \%$ of cases, was pulmonary restrictive disease. Indications of NIV were acute exacerbations in patients with chronic domiciliary NIV in three patients, hypoxic ARF in six and hypercapnic ARF in five. The diagnoses were pneumonia/atelectasis in seven patients, bilateral extensive pneumonia in three, RSV bronchiolitis in two, apnea in one, and asthma exacerbation in one. Only one patient required intubation for mechanical ventilation, all others improved. The procedures did not have complications. NIV lasted less than three days in 5 patients, 4 to 7 days in four patients and more than 7 days in five. One third of the patients required fiberoptic bronchoscopy for massive or lobar atelectasis and one third remained on domiciliary NIV program. Conclusions: NIV can be useful and safe in children with ARF admitted to a Pediatric Intermediate Care Unit. If strict inclusion protocols are followed, NIV might avoid mechanical ventilation (Rev Méd Chile 2005; 133: 525-33).

(Key Words: Continuous positive airway pressure; respiratory insufficiency, Ventilation)

Recibido el 10 de noviembre, 2004. Aceptado en versión corregida el 17 de marzo, 2005.

${ }^{1}$ Departamento de Pediatría, Campus Centro, Facultad de Medicina, Universidad de Chile.

${ }^{2}$ Unidad de Intermedio Médico-Quirúrgico, Servicio de Pediatría, Complejo Hospitalario San Borja Arriarán.

${ }^{3}$ Unidad de Respiratorio Infantil, Complejo Hospitalario San Borja Arriarán. Santiago de Chile.

Correspondencia a: Dr. Francisco Prado A. Unidad de Respiratorio Infantil. Vasco Núñez de Balboa 1355, Las Condes, Santiago, Chile. Tel: 56-2-2281000. Fax: 56-2-5446375. Cel: 092363052. E mail: panchoprado2004@yahoo.com 
$\mathrm{L}$ a asistencia ventilatoria no invasiva (AVNI) es una modalidad de ventilación mecánica con presión positiva, entregada por una mascarilla nasal o facial ${ }^{1}$. De esta manera, puede evitar las complicaciones derivadas de la intubación de la vía aérea, traqueostomía y ventilación mecánica convencional (VMC).

El sistema, más frecuentemente, utiliza generadores de flujo y presión positiva en 2 niveles (BiPAP), como asistencia ventilatoria no invasiva domiciliaria (AVNID), a objeto de aumentar la capacidad residual funcional (CRF) y disminuir el trabajo respiratorio en pacientes con patologías restrictivas, principalmente por enfermedades neuromusculares ${ }^{2,3}$ y xifoescoliosis $^{4}$ que determinan insuficiencia primaria de la bomba respiratoria. Se utiliza también en niños con fibrosis quística ${ }^{5,6}$, síndrome de hipoventilación cen$\operatorname{tral}^{7}$, apnea obstructiva del sueño ${ }^{8}$ y obstrucción de la vía aérea superior' ${ }^{9}$. La AVNI es una alternativa en pacientes con insuficiencia respiratoria aguda grave (IRAG), que estén en vigilia y tengan una vía aérea estable $10-12$.

En niños con insuficiencia respiratoria aguda, logra los mismos beneficios que en adultos, mejorando la ventilación y oxigenación, disminuyendo el trabajo respiratorio y probablemente evitando la necesidad de $\mathrm{VMC}^{1,12}$. En niños asmáticos con exacerbaciones agudas ha demostrado resultados similares a los observados en pacientes adultos ${ }^{13,14}$. En Unidades de Cuidado Intensivo Pediátricas (UCIP), permite la salida precoz de VMC, acortando los tiempos de intubación ${ }^{15}$ y en pacientes de alto riesgo anestésico, entrega soporte ventilatorio durante procedimientos invasivos realizados con sedación ${ }^{16}$. En cuidado intensivo neonatal, el uso de cPAP y ventilación con presión positiva nasal, permite la extubación precoz, evita atelectasias y acorta la VMC, disminuyendo el riesgo de enfermedad pulmonar crónica y estenosis subglótica ${ }^{17}$.

En el año 2001, presentamos nuestra experiencia con AVNI utilizando generadores de flujo y mascarillas nasales para el tratamiento de la IRAG en una Unidad de Intermedio Pediátrico (UIP) ${ }^{18}$.

El objetivo de esta publicación es evaluar los resultados actuales de la AVNI, 8 años después de introducir esta modalidad de ventilación mecánica como terapia usual para niños con IRAG en una UIP.

\section{MÉTODO}

Se revisaron las fichas clínicas de los pacientes ingresados a UIP del Complejo Hospitalario San Borja Arriarán sometidos a un protocolo de AVNI por IRAG, ya sea con fallo respiratorio hipóxico agudo o insuficiencia respiratoria aguda tipo II, pero sin acidosis respiratoria, desde el primero de enero al primero de octubre de 2004. Se definió fallo respiratorio hipóxico agudo ${ }^{12}$ a los requerimientos de $\mathrm{FiO}_{2}>0,4$ para lograr $\mathrm{SaO}_{2}>90 \%$, más la aparición de signos clínicos de aumento del trabajo respiratorio. Los criterios de selección de los pacientes se detallan en la Tabla 1. La decisión de ingreso a AVNI o traslado a UCIP se tomó dentro de las primeras $36 \mathrm{~h}$ de evolución en la UIP.

\section{Tabla 1. Asistencia ventilatoria no invasiva en insuficiencia respiratoria aguda - Indicaciones}

\begin{tabular}{|ll|}
\hline Criterios de inclusión" & Criterios de exclusión \\
\hline Insuficiencia respiratoria aguda con: & \\
- Aumento del trabajo respiratorio y & - Inestabilidad hemodinámica \\
frecuencia respiratoria $>$ al límite & - Compromiso agudo de conciencia \\
superior para la edad & - Neumotórax o neumomediastino \\
- $\mathrm{FiO}_{2}>0,4$ y saturación de & - Indicación de intubación: \\
hemoglobina $<93 \%$ & - Acidosis respiratoria por $\mathrm{pH}<7,25$ \\
$\quad$ y/o & $-\mathrm{SaO}_{2}<90 \%$ con FiO $\mathrm{F}_{2}>0,6 \mathrm{O} \mathrm{Pa}_{\mathrm{FiO}}<100$ \\
- Hipercapnia con pH no inferior a 7,25 & - Vía aérea inestable \\
- Progresión del compromiso & - Falla orgánica múltiple \\
pulmonar en Rx de tórax &
\end{tabular}

"Indicación precoz en pacientes con enfermedad neuromuscular o xifoescoliosis toracoabdominal. 
Se consideró éxito la mejoría clínica, definida como: a) resolución de la patología que motivó la indicación de AVNI, con egreso de la UIP sin soporte ventilatorio o b) resolución de la exacerbación aguda en pacientes crónicos, logrando volver a los parámetros basales de AVNID o ingresar a ella una vez estabilizados. Se consideró fracaso la progresión de la insuficiencia respiratoria o necesidad de traslado a UCIP para VMC, intolerancia a la mascarilla nasal o a los flujos entregados por más de una hora, u otra complicación que imposibilitara la continuidad de la terapia (neumotórax, neumomediastino).

Se usó BiPAP (Respironics Inc modelo Bipap S/T-D, Nellcor Puritan Benett modelo Knigth Star 335) y mascarillas nasales siliconadas (Figura 1), según el tamaño de la cara del paciente (Respironics, Taema modelo Concept 5 y Resmed modelo Protegé o Ultramirage). En lactantes menores de un año se utilizó mascarilla nasal Resmed Infants. La oxigenoterapia necesaria para $\mathrm{SaO}_{2}$ igual o superior a 90\%, se suministró con bolsa reservorio y válvula unidireccional adaptada por medio de conexión en $\mathrm{T}$ al corrugado del circuito, más humidificador de burbuja (Figura 2).
Inicialmente, se fijó la sensibilidad en valor mínimo, hasta lograr buena tolerancia con presión positiva inspiratoria (iPAP) más presión positiva espiratoria (ePAP) de 10 y $5 \mathrm{~cm}$ de $\mathrm{H}_{2} \mathrm{O}$, aumentando en forma individual cada una de ellas para disminuir la $\mathrm{PaCO}_{2}$ (iPAP) o mejorar la $\mathrm{SaO}_{2}$ (ePAP). Se mantuvo presiones diferenciales iguales o mayores de $6 \mathrm{~cm}$ de $\mathrm{H}_{2} \mathrm{O}$ para lograr un volumen corriente espirado de aproximadamente $10 \mathrm{ml} \mathrm{x} \mathrm{kg}$. No se indicó iPAP $>20 \mathrm{~cm}$ de agua y ePAP $>10 \mathrm{~cm} \mathrm{H}_{2} \mathrm{O}$; los pacientes con necesidades mayores a las descritas se trasladaron a UCIP. En modalidad A/C se fijó FR mínima de $10 \mathrm{x}$ min. La AVNI continua se limitó a 48 h, para luego alternar 2 ó 3 ciclos de $3 \mathrm{~h}$ diurnos, más ciclo nocturno de $10 \mathrm{~h}$ (22 PM a 8 AM). Como el flujo inspiratorio en los lactantes puede ser insuficiente para activar el umbral de los equipos, utilizamos en ellos BiPAP Respironic ST/D en modalidad controlada con frecuencia respiratoria (FR) de 30 por min y presión diferencial no menor de $5 \mathrm{cms}$ de agua, para capturar el esfuerzo respiratorio del paciente.

Se utilizaron monitores Nellcor Puritan Bennett para registro simultáneo de $\mathrm{SaO}_{2}$, presión arterial, frecuencia cardíaca (FC), FR e impedancia toráci-

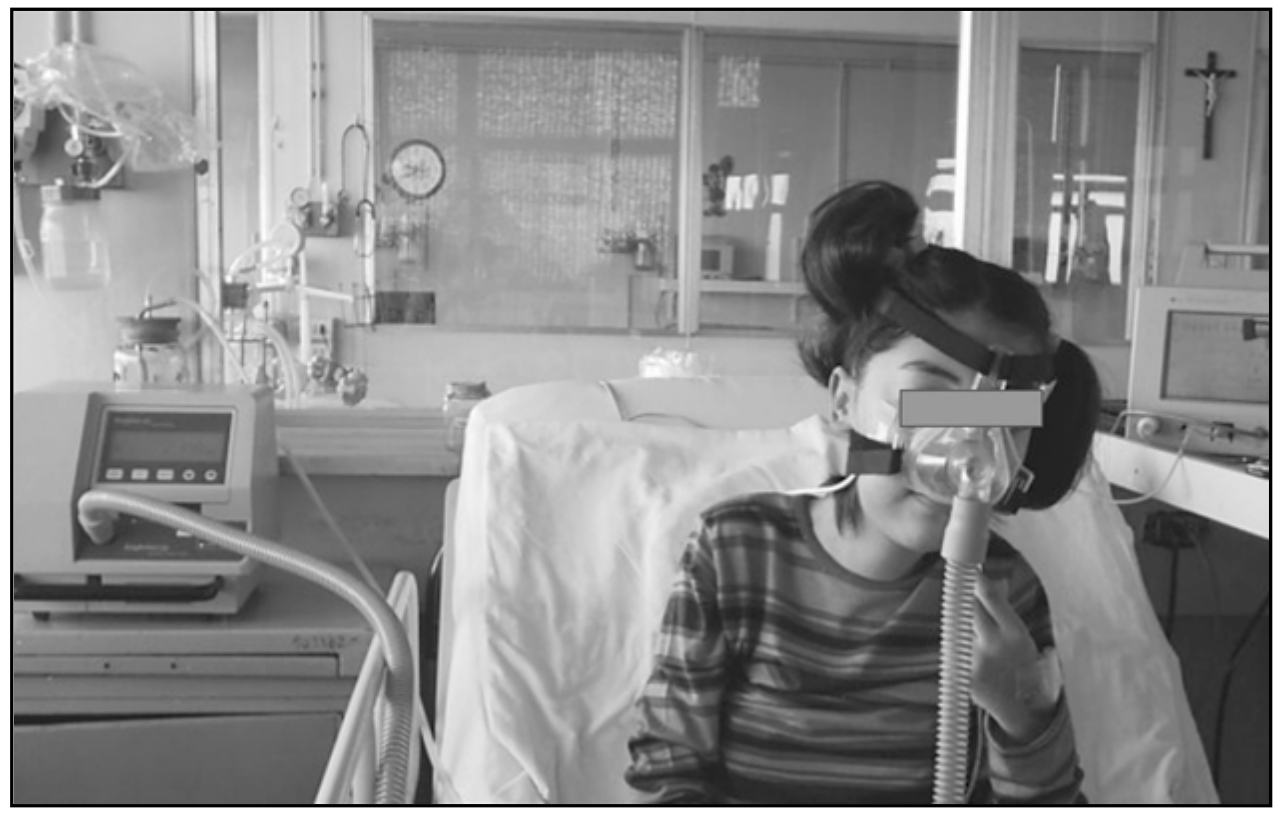

FIgURA 1. Mascarilla nasal y arnés de fijación. 


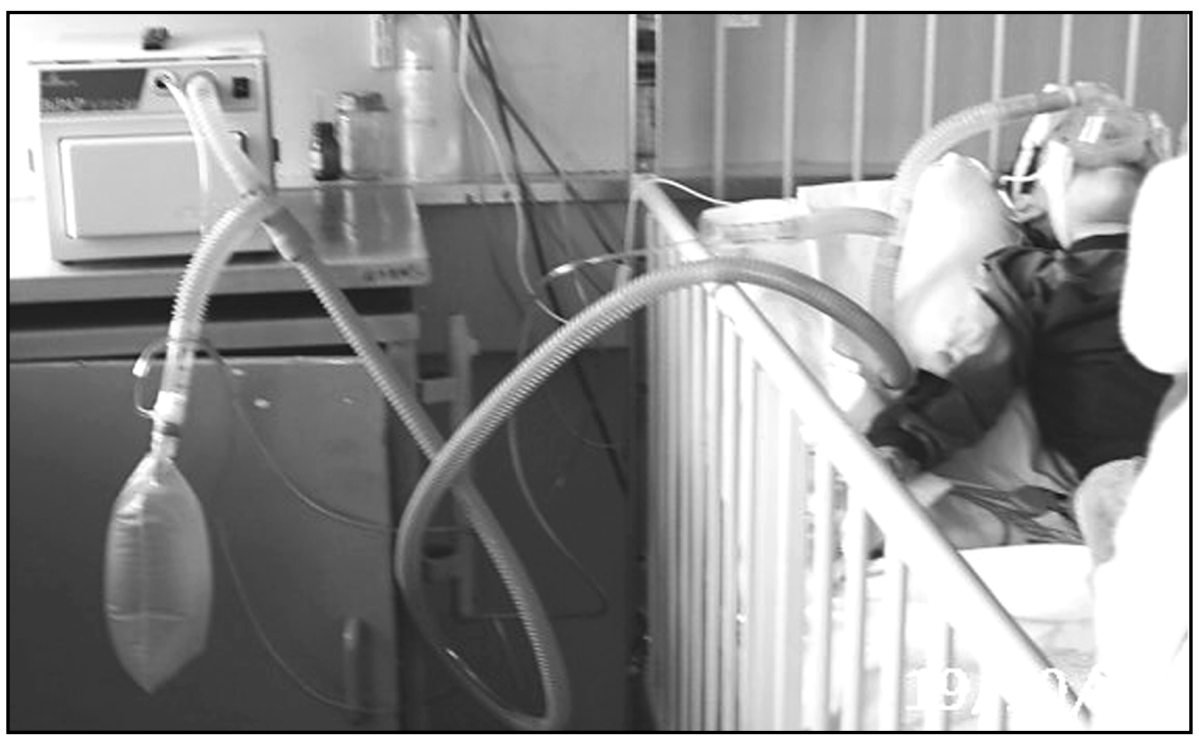

FIgura 2. Dispositivo para oxigenoterapia. Reservorio de mascarilla de alto flujo y conexión en T a corrugado del circuito de ventilación.

ca. Se vigilaron los puntos de apoyo de la mascarilla sobre la cara, cuidando que no existiera escape de aire hacia los ojos y evitar la distensión gástrica, si ocurría, se indicaba ayuno y sonda naso-gástrica a caída libre.

Se tomaron muestras de gases en sangre arterial (GSA) antes de la conexión y luego de $60 \mathrm{~min}$ de lograda la adaptación a la mascarilla nasal. El control posterior dependió de la condición de cada paciente. Dentro de las primeras 24 h, se tomó radiografía de tórax portátil con el objeto de evaluar volúmenes pulmonares y cambios respecto al basal. En caso de atelectasias lobares o masivas, se realizó fibrobroncoscopia (FBC), con fibrobroncoscopio Olympus 3C30 vía transnasal, colocando lidocaína al $2 \%$ en narina de acceso, como anestesia tópica, y sedación con midazolam intravenoso 0,25 mg por kilo de peso. Criterios de retirada de AVNI: a) mejoría de la condición clínica con resolución de apnea, disminución de la disnea, aumento de la fuerza muscular y mejoría de la oxigenación con $\mathrm{SaO}_{2}>93 \%$ con $\mathrm{FiO}_{2}<0,4$; b) mejoría del trabajo respiratorio evaluado por disminución de la FR con onda de impedancia torácica sinusoidal y normalización de la FC; c) mejoría en índices de ventilación con eucapnia en el paciente sin insuficiencia respiratoria crónica (IRC) y $\mathrm{PaCO}_{2}$ en rango de su nivel histórico para aquellos pacientes hipercápnicos; d) mejoría radiológica con resolución atelectasia, disminución o desaparición de imágenes parenquimatosas pulmonares y, a lo menos, 7 espacios intercostales en ambos campos pulmonares y e) regreso a parámetros habituales de ventilación y condición médica estable, en los pacientes en AVNID.

Los pacientes que no cumplieron estos requisitos, fueron considerados para AVNID. El retiro de AVNI fue progresivo, disminuyendo la FR hasta dejar en asistida, luego iPAP hasta igualar con ePAP. Por último, se disminuyó cPAP hasta $4 \mathrm{~cm}$ de agua y se continuó con períodos de desconexión progresiva. Se mantuvo oxigenoterapia, según necesidad, para mantener $\mathrm{SaO}_{2}>93 \%$.

\section{Resultados}

En el período estudiado, se ingresaron a la UIP 300 pacientes con insuficiencia respiratoria aguda, 49 evolucionaron dentro de las primeras $36 \mathrm{~h}$ con IRAG (16\%), de ellos, 14 (29\%) fueron sometidos al protocolo descrito de AVNI y 35 (71\%) fueron 
trasladados a UCIP por presentar criterios de mayor gravedad. Se disponía de 5 generadores de flujo en una UIP con 10 cupos.

Ocho pacientes eran hombres, la edad promedio fue 6,2 años, con rango de 1 mes a 13 años. Las características clínicas se muestran en la Tabla 2, las indicaciones, trastorno fisiopatológico a corregir y evolución, en Tabla 3.

Cuatro pacientes eran sanos previo al ingreso y 10 tenían comorbilidad. Siete de ellos tenían enfermedad neuromuscular, parálisis cerebral o trastorno del centro respiratorio con fallo primario de la bomba respiratoria, agravada en 6 por xifoescoliosis severa. La AVNI se indicó mayoritariamente por trastorno ventilatorio restrictivo secundario a neumonía y atelectasia. Tres pacientes crónicos que estaban en AVNID se hospitalizaron por descompensación aguda. El resto de los pacientes ingresó por insuficiencia respiratoria aguda sin hipoventilación crónica de base, salvo uno con
Tabla 2. Pacientes tratados con AVN I: características clínicas

\begin{tabular}{|lr|}
\hline Número pacientes & 14 \\
Sexo (M:H) & $6: 8$ \\
Edad & \\
1 mes a 13 años & 3 \\
$<1$ a: & 3 \\
$2-5 \mathrm{a}:$ & 8 \\
$6-15 \mathrm{a}:$ & 10 \\
Comorbilidad & \\
$\quad$ Respiratoria & 1 \\
$\quad$ Asma bronquial & 1 \\
$\quad$ DPC & 1 \\
Cardiopatía congénita & \\
$\quad$ Ventrículo único & 1 \\
Neurológica & \\
$\quad$ Hipoventilación central/Sínd. diencefálico & \\
$\quad$ Miopatía congénita: & 1 \\
$\quad$ A. espinal tipo II & 1 \\
$\quad$ Miopatía Ulrich & 3 \\
$\quad$ Parálisis cerebral & 1 \\
$\quad$ Sínd. hipotónico & \\
\hline
\end{tabular}

DPC: Daño pulmonar crónico.

Tabla 3. Pacientes tratados con AVN I: Indicaciones, modalidad y evolución

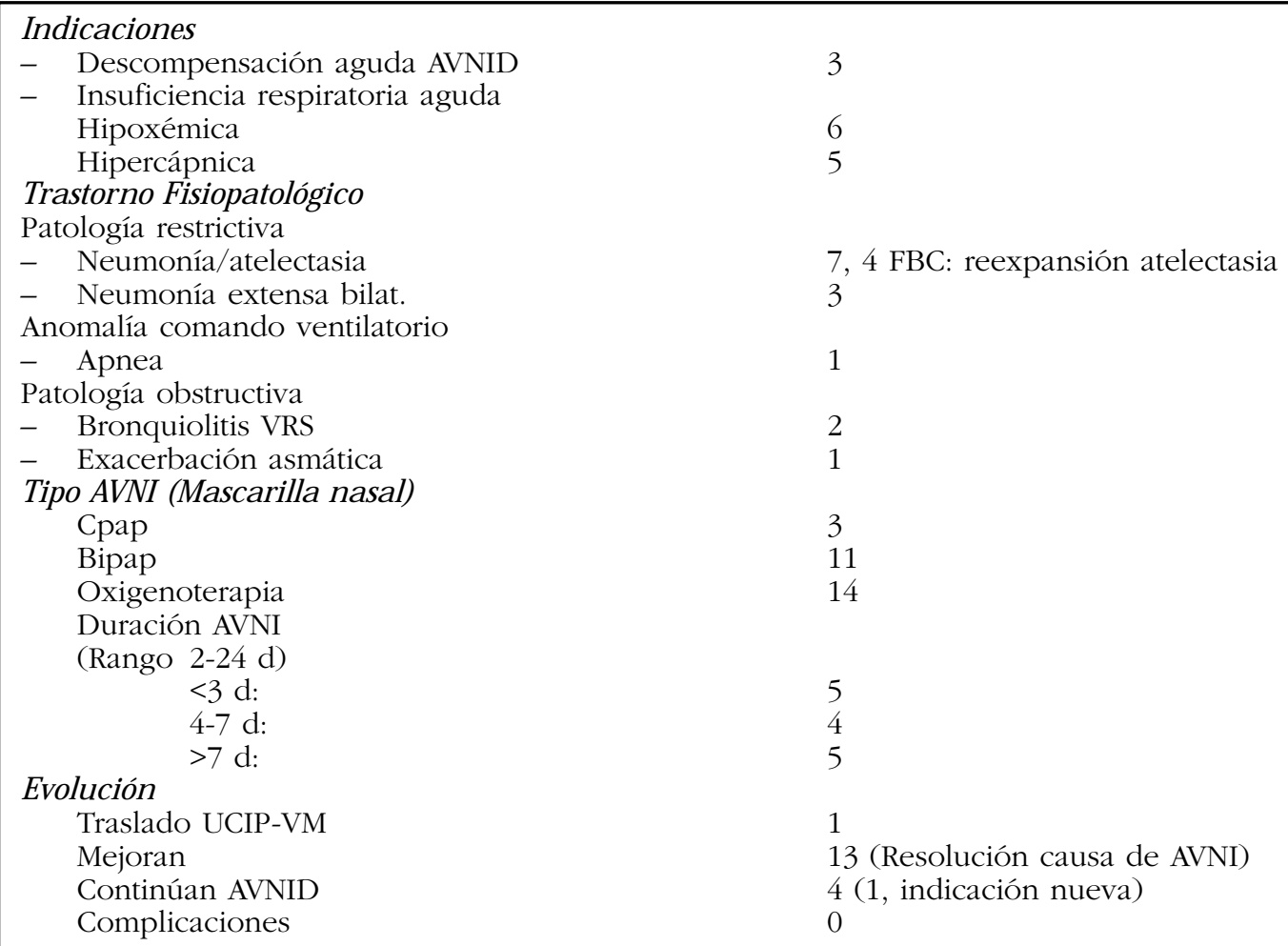

AVNID: asistencia ventilatoria no invasiva domiciliaria, VM: ventilación mecánica, UCIP: Unidad de cuidado intensivo pediátrico. FBC: Fibrobroncoscopia. 
miopatía congénita que después continuó con AVNID. Ocho de ellos presentaron hipercapnia. Sólo 3 pacientes ingresaron por obstrucción de la vía aérea baja secundaria a bronquiolitis y exacerbación asmática. Todos los niños tuvieron hipoxemia secundaria, con grados variables de trastornos de ventilación-perfusión, se logró mantener una apropiada $\mathrm{SaO}_{2}$ con un sistema ad-hoc de oxigenoterapia. Cuidando no ocluir los lugares de exhalación de las mascarillas, no hubo recirculación de CO2 e hipercapnia secundaria. En cuatro pacientes, se hizo FBC para reexpandir atelectasias masivas o lobares (Figura 3). La AVNI se entregó preferentemente con BiPAP (79\%) y tuvo una duración inferior a 7 días en $2 / 3$ de los pacientes, fue exitosa resolviendo la causa que motivó su indicación en 13 pacientes y no se observaron complicaciones. Sólo una niña de 13 años, con miopatía de Ulrich, necesitó intubación endotraqueal y VMC; al quinto día, se le realizó FBC por atelectasia masiva e inmediatamente posterior al procedimiento se logró pasar a AVNI con reexpansión pulmonar casi completa (Figura 4).

\section{DisCUSIÓN}

En una UIP, con criterios de selección estrictos, la AVNI implementada con generadores de flujo y mascarilla nasal puede ser eficiente y segura en la mejoría clínica de los pacientes con IRAG. En nuestros pacientes permitió mejorar el trabajo respiratorio, evaluado por la disminución de la disnea, FR y generación de curvas de impedancia torácica sinusoidales que acompañó la mejoría en la oxigenación, ventilación y la estabilidad o resolución de la patología que motivó la asistencia ventilatoria.

En esta revisión no insistimos en la tabulación de parámetros gasométricos o del trabajo respiratorio, objetivo que fue valorado por nuestro grupo en una publicación anterior ${ }^{18}$. En ella observamos que la AVNI logró significativamente disminuir la $\mathrm{PaCO}_{2}$, FR y tendió a normalizar el $\mathrm{pH}$ y disminuir la $\mathrm{FiO}_{2}$ necesaria para mantener $\mathrm{SaO}_{2}>93 \%$ dentro de las primeras $6 \mathrm{~h}$ de su inicio. Los resultados clínicos presentados son concordantes con otros estudios pediátricos en que la AVNI se utilizó en pacientes con insuficiencia respiratoria aguda, especialmente en aquellos con patología pulmonar restrictiva crónica, hipoventilación nocturna y descompensaciones agudas. En nuestra experiencia, como en las publicaciones citadas, la mayoría de estas descompensaciones se deben a neumonías y atelectasias $1,3,10-12$. Casi 50\% de nuestros pacientes tenían xifoescoliosis severa, secundaria a miopatías o parálisis cerebral. La disminución de la distensibilidad toracoabdominal

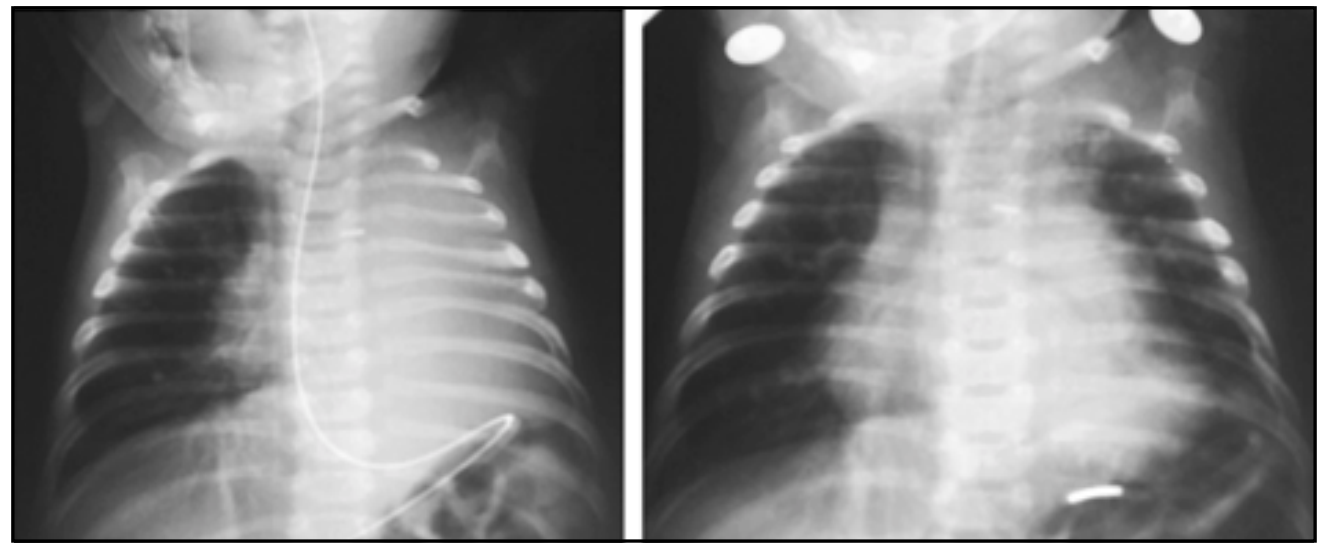

Figura 3. Lactante de 2 meses con ventrículo único operado y broncomalacia del bronquio fuente izquierdo. Atelectasia masiva izquierda reexpandida luego de FBC e inicio AVNI. 


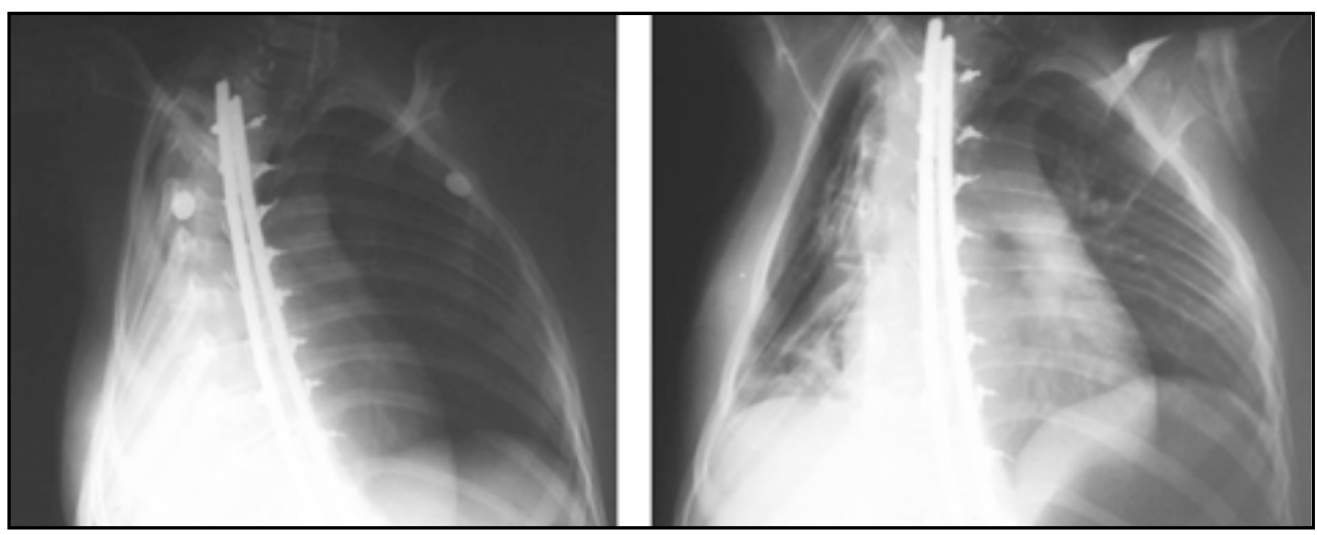

Figura 4. Atelectasia masiva en paciente con miopatía de Ulrich, control radiológico luego de FBC y una semana de AVNI.

derivada de esta condición ${ }^{19}$, se asocia a un importante aumento del índice tensión/tiempo de los músculos respiratorios ${ }^{20}$, disminuyendo el umbral de fatiga frente a la sobrecarga de trabajo por infecciones o atelectasias. La respuesta favorable a la AVNI es bien conocida en estos pacientes $^{21,22}$ y debería considerarse precozmente en la evolución de una descompensación aguda.

La decisión de revisar el período actual de AVNI, fue para evaluar los resultados de un protocolo con criterios de selección y acciones planificadas, en forma prospectiva en una UIP a plena capacidad, para entregar esta terapia en un amplio rango de edad incluyendo lactantes $<1$ año, condición más favorable al período inicial reportado en $2001^{18}$. Esto permitió incorporar un número similar de pacientes en un corto tiempo y desplazar su indicación a niños menores. Dado el escenario en que se implementó la AVNI, no fue posible evaluar su indicación como alternativa a la VMC, esto nos llevó a definir criterios precisos para las 2 opciones de soporte ventilatorio ${ }^{1,12}$. Del total de pacientes con IRAG, un tercio recibió AVNI y el resto requirió traslado a UCIP para VMC. El grupo de pacientes tratados con AVNI tenía significativo aumento del trabajo respiratorio, con hipoventilación o hipoxemia y en ellos, nos pareció que existía clara indicación de apoyo ventilatorio como para mantener un grupo control sólo con tratamiento convencional. Sin existir consenso, la AVNI parece recomendable en aquellos pacientes con insuficiencia respiratoria hipercápnica (Tipo II), más aún si existe compromiso primario de la bomba por enfermedad neuromuscular y xifoescoliosis. En aquellos pacientes con IRAG tipo I, la oxigenoterapia es el principal tratamiento, sin embargo, cuando existe significativo aumento del trabajo respiratorio y $\mathrm{FiO}_{2}>0,4$, la AVNI puede ser considerada ${ }^{1,12}$. Sin existir indicaciones absolutas, nos parece que los criterios de selección utilizados minimizan la sobreutilización de esta técnica.

Aun cuando las conclusiones inferidas tienen la limitación de ser una serie clínica y un reporte descriptivo sin grupo control, la mayoría de nuestros pacientes tenía un trastorno ventilatorio restrictivo cuyo manejo con AVNI está bien documentado ${ }^{10}$. Esta indicación, menos controvertida, se ha extendido a la IRAG, incluso en niños con asma aguda sin comorbilidad de la bomba respiratoria ${ }^{13,14}$. Sin embargo, faltan trabajos prospectivos, controlados y randomizados que permitan validar estos resultados. En patología obstructiva, como bronquiolitis, la experiencia se limita a reportes de casos clínicos o series pequeñas con nCPAP 23,24 .

En 93\% de nuestros pacientes hubo mejoría, resultado comparable a lo publicado por otros 
autores ${ }^{10,11}$. El alto porcentaje de resolución de neumonías y atelectasias, probablemente, guardan relación con la mejoría de la ventilación y reexpansión de unidades alveolares colapsadas luego del aumento de la CRF. El uso precoz de fibrobroncoscopia en un tercio de los pacientes, permitió la rápida reexpansión de atelectasias masivas o lobares.

En el paciente con miopatía congénita en que la AVNI fracasó, es muy probable que la utilización de dispositivos de tos asistida hubiera facilitado el tratamiento fuera de $\mathrm{VMC}^{25}$.

No hubo complicaciones derivadas de la AVNI, la más frecuente de ellas, descrita en $20 \%$, es el edema del puente nasal ${ }^{1}$. Es probable que limitar la AVNI continua, con paso precoz a ciclos diurnos y nocturnos, más medidas simples de enfermería como el uso de Tegaderm en sitios de apoyo de la mascarilla y la vigilancia de una buena adaptación que minimice flujos de escape, sea responsable de estos resultados. La dificultad para tener mascarillas nasales adecuadas al tamaño de la cara del paciente, la utilización de equipos que entregan presión a flujos elevados (generadores de flujos con turbina) y presiones diferenciales limitadas por iPAP inferiores a $25 \mathrm{~cm}$ de agua entorpecen la AVNI en niños pequeños y en otros con IRAG hipoxémica. No obstante, en 3 lactantes menores de un año, 2 de ellos con bronquiolitis y otro con neumonía atelectásica y cardiopatía compleja, se logró establecer con

\section{REFERENCIAS}

1. NøRRegaARd O. Noninvasive ventilation in children. Eur Respir J 2002; 20: 1332-42.

2. Mellies U, Ragette R, Dohna SC, Boehm H, Voit T, Teschler H. Long-term noninvasive ventilation in children and adolescent with neuromuscular disorders. Eur Respir J 2003; 22: 631-6.

3. Birnkrant DJ, Pope JF, Eiben RN. Noninvasive management of pediatric neuromuscular ventilation failure. J Child Neurol 1999; 14: 139-43.

4. Simonds AK, Wards S, Heather S, Bush AB. Outcome of paediatric domiciliary mask ventilation in neuromuscular and skeletal disease. Eur Respir J 2000; 16: 476-81 éxito. El uso de una mascarilla nasal especialmente diseñada para lactantes y BiPAP en modalidad control, ha sido utilizada exitosamente en lactantes con patologías restrictivas y mixtas ${ }^{23,26}$.

Este protocolo de AVNI en UIP, podría ser establecido en otros centros, racionalizando el ingreso a unidades de camas críticas. La necesidad de un tercio de los pacientes de permanecer con AVNID, refuerza la importancia de contar con programas complementarios ${ }^{27-29}$ que permitan liberar precozmente el recurso existente para manejo intrahospitalario. La UIP es el escenario ideal para la transición hacia dichos programas.

Probablemente, en un futuro cercano el uso de equipos con mayor sensibilidad a pequeños esfuerzos respiratorios, mayor versatilidad en la entrega de presión positiva en 2 niveles y en las interfases de conexión, permita el uso rutinario de la AVNI como alternativa para el tratamiento de la IRAG en niños pequeños, tanto con patología obstructiva y restrictiva, extendiendo su uso en distintos escenarios no sólo en UCIP.

Sugerimos que la AVNI con protocolos estrictos y criterios seleccionados de inclusión, usada con generadores de flujo, mascarillas nasales y dispositivos modificados para oxigenoterapia convencional en pacientes con IRAG y una vía aérea estable puede ser utilizada en forma eficiente y segura en una UIP, pudiendo evitar la ventilación mecánica convencional.

5. Brigitte F, Boule M, Lofaso F, Zerah F, Cleiment A, Harf A ET AL. Chest physiotherapy in cystic fibrosis: improved tolerance with nasal pressure support ventilation. Pediatrics 1999; 103: 32.

6. Hodson ME, Madden BP, Steven MH, Tsang VT, YACOUB MH. Noninvasive mechanical ventilation for cystic fibrosis, a potentional bridge to transplantation. Eur Respir J 1991; 4: 524-7.

7. Villa MP, Dotta A, Castello D, Piro S, Pagani J, Palamadis S ET AL. Bi-Level positive airway pressure (BIPAP) ventilation in an infant with central hypoventilation syndrome. Pediatr Pulmonol 1997; 24: 66-9.

8. McNamara F, Suluivan C. Obstructive sleep apnea in infants and its management with nasal continuos 
positive airway pressure. Chest 1999; 116: 10-6.

9. Teague WG, Kervin LJ, Diwadkar UV, Scott PH. Nasal bi-level positive airway pressure (BIPAP) acutely improves ventilation and oxigen saturation in children with upper airway obstruction (abstr). Am Rev Respir Dis 1991; 143: A505.

10. Fortenberry JD, Del Toro J, Jefferson LS, Evey L, HaAse D. Management of pediatric acute hypoxemic respiratory insufficiency with bilevel positive pressure (BiPAP) nasal mask ventilation. Chest 1995; 108: 1059-64.

11. Padman R, Lawless ST, Kettrick RG. Noninvasive ventilation via bilevel positive airway pressure support in pediatric practice. Crit Care Med 1998; 26: $169-73$.

12. Teague WG. Noninvasive ventilation in the pediatric intensive care unit for children with acute respiratory failure. Pediatr Pulmonol 2003; 35 : 418-26.

13. Thill PJ, McGuire JK, Baden HP, Green TP, Checchia PA. Noninvasive positive-pressure ventilation in children with lower airway obstruction. Pediatr Crit Care Med 2004; 5: 337-42.

14. Akingbola OA, Simakajornboon N, Hadley Jr EF, Hopkins RL. Noninvasive positive-pressure ventilation in pediatric status asthmaticus. Pediatr Crit Care Med 2002; 3: 181-4.

15. Pope JF, Birnkrant D, Besunder J, Martin Jurmetz K, Buildt D. Noninvasive nasal ventilation can facilitate endotracheal extubation in the pediatric intensive care unit. Chest 1996; 11: 110: 184-5.

16. Pope J, Birnkrant D, Martin J, Repucci A. Noninvasive ventilation during percutaneous gastrostomy placement in Duchenne muscular dystrophy. Pediatric Pulmonol 1997; 23: 468-71.

17. Halliday HL. What interventions facilitate weaning from the ventilator? A review of the evidence from systematic reviews. Paediatr Respir Rev 2004; (suppl A): S347-52.

18. Prado F, Boza ML, Godoy ma, Koppmann A, Guillén B, Tejerina H. Asistencia ventilatoria no invasiva con sistemas generadores de flujo en pediatría, experiencia 1996-1999. Rev Chil Enf Resp 2001; 17: 10-8.
19. Papastamelos C, Panitch HB, Allen Jl. Chest wall compliance in infants and children with neuromuscular disease. Am J Respir Crit Care Med 1996; 154: 1045-8.

20. Mulreany LT, Weiner DJ, McDonough JM, Panitch HB, Allen JL. Noninvasive measurement of the tension-time index in children with neuromuscular disease. J Appl Physiol 2003; 95: 931-7.

21. Ferris G, Servera-Pieras E, Vergara P, Tzeng aC, Pérez M, Marin J ET al. Kyphoscoliosis ventilatory insufficiency: non invasive management outcomes. Am J Phys Med Rehabil 2000; 79: 24-9.

22. Eluis ER, Grustein RR, Chan S, Bye PT, Sullivan CE. Non Invasive ventilatory support during sleep improves respiratory failure in kyphoscoliosis. Chest 1988; 94: 811-5.

23. Vega-Briceño L, Contreras i, Sánchez I, Bertrand P. Uso precoz del BiPAP en el tratamiento de la insuficiencia respiratoria en un lactante con osteogénesis imperfecta. Caso clínico. Rev Méd Chile 2004; 132: 861-4.

24. Soong WJ, Hwang B, Tang RB. Continuous positive airway pressure by nasal prongs in bronchiolitis. Pediatr Pulmonol 1993; 16: 163-6.

25. Miske LJ, Hickey EM, Kolb SM, Weiner DJ, Panitch HB. Use of the mechanical in-exsufflator in pediatric patients with neuromuscular disease and impaired cough. Chest 2004; 125: 1406-12.

26. Bach J, Niranjan V, Weaver B. Spinal muscular atrophy type I. A noninvasive respiratory management approach. Chest 2000; 117: 1100-5.

27. Prado F, Boza Ml, Koppmann A. Asistencia ventilatoria no invasiva domiciliaria nocturna en pediatría. Rev Chil Enf Respir 2003; 19: 146-54.

28. Martínez Carrasco C, Barrio Gómez de Agüero I, Antelo Landeiro C. Nasal mechanical ventilation in pediatric patients at home. An Esp Pediatr 1997; 47: 269-72.

29. Sánchez i, Valenzuela A, Bertrand P, Alvarez C, Holmgren N, Vilchez S et al. Apoyo ventilatorio domiciliario en niños con insuficiencia respiratoria crónica. Experiencia clínica. Rev Chil Pediatr 2002; 73: 51-5. 